\title{
Health and Safety in the Learning Environment
}

BY SIMON BOXALL

Before I start, let me state that I have great respect for health and safety-both at work and at home-and that maintaining a safe learning environment for students is paramount. This is particularly true for oceanography, where we work with some of the nastier chemicals and with high voltages, all in a boat on a stormy, deep, unstable sea. Let's face it: if we were to initiate the subject of oceanography from scratch today, it would be banned. But, are we being overcautious to a point where a sense of true risk is no longer being instilled into our new generations of scientists? Is the purpose of health and safety moving from a pragmatic approach to the issue to a paper exercise that not only takes logic away but is more focused on litigation rather than avoiding mishaps?

The purpose of assessing risk is to ensure that any individual carrying out work is fully aware of the hazards associated with a task, and that precautions are taken to reduce or, ideally, remove risk. So far, so good. But, do students learn how to identify true risk and how to minimize impact, especially when things do (and they will) go wrong?

\section{Simon Boxall (srb2@noc.soton.}

ac.uk) is Associate Fellow, University of Southampton, National Oceanography Centre, Southampton, UK.
In my $\mathrm{PhD}$ training days (a couple or so years ago), we were in a small department where postdocs worked together in the field by themselves. This situation often meant taking a small RIB (rigid inflatable boat) off into the coastal region of the Mersey River to collect samples. We were responsible for getting there, for sampling, and for safety (i.e., getting back in one piece). Two of us were experienced boat handlers, and we had to go through training with the chief technician. We had radios that we checked and double-checked, up-to-date flares, and lifejackets. Over the numerous "voyages," we encountered and overcame a variety of hazards that we could never have assessed on paper beforehand. These hazards ranged from encountering a hidden hydraulic jump that swamped the RIB, to being stranded on a mud bank for five hours (the up-to-date charts omitted its presence), to recovering a colleague who lost his footing and ended up in the Mersey. At that time, I'm not sure if drowning or chemical poisoning was the higher risk. The one we never reported was the embarrassment of getting the Land Rover stuck up to its chassis in the mud in the intertidal zone-its amazing what you can do with a bulldozer borrowed from a local building site and a car wash. The point is that in all cases we were equipped to deal with hazards and learned how to avoid them.

To do the same now, we would have to take formal courses in boat handling, first aid, sea survival, and VHF radio operation, and have a medical exam. This education is good, surely? No, not really. First aid and sea survival are short courses that don't have a pass or fail element-you just have to be there. As for boat handling and VHF radio? The first is a long weekend course, the latter half a day. One can go from complete novice to qualified in a short period, and on the courses I did in the past, no one failed (and, believe me, some should have). What is worse is that once we have given our students this experience, they are set up for life. I do have to do a very useful refresher course on first aid every three years, but my sea survival and VHF classes are one-offs, even if I don't practice them for years at a time.

Armed with all the safety kits and certificates we can accrue, we think we become immune to danger. I am aware of apocryphal tales of children suffering more injuries now that playgrounds are rubber-lined, slow-moving safety zones-their ability to recognize danger has not been learned though experience. As a scientist, I had to try to find the truth behind this assertion. The library at the National Oceanography Centre (NOC), Southampton, is great 
for marine science but poor on social science, so I resorted to Google Scholar. Typing in "risk perception in student fieldwork" produced a significant number of learned articles on students' interpretations on the risks of sexual activity, in the field and the classroom. Perhaps we need another section in our own Risk Assessment Form in the future!

Slovic (1987) is often attributed as one of the original proponents of what our social science colleagues call the "psychometric paradigm." He provides evidence that those who oversee health and safety issues need to take account of how the target audience perceives and responds to risk. Having safety imposed doesn't work; having it embedded in one's practice and experience does. Although there is an array of papers that subsequently applies this paradigm to risks of certain types of medical treatments, to how people deal with earthquakes, few deal with students' perceptions. Most, such as Cook et al. (2006), deal with how educational staff members are stopping fieldwork because of their perceptions of how the potential litigation associated with such activity exposes their careers, finances, and senses of wellbeing.

My informal upbringing in health and safety has equipped me to deal with a wide variety of situations, making (I hope) sensible and well-guided assessments of hazard. In oceanography, we have to work all over the world with a diversity of cultures and approaches to risk. Few are as prescriptive or carry as significant a paper trail as in Europe or the United States-and even within Europe, what is considered risky varies. As a professional, I need to assess the true hazards and either minimize them to an acceptable level, or stop. That approach takes continual experience and vigilance-experiences that were first learned from working alongside peers and tutors in my student days, or even from playing as a child on the concrete playground. If we replace a sensible and pragmatic approach to minimizing hazards with reams of paper and sometimes meaningless qualifications, then we have a problem ahead. Our students won't be equipped to work in the field, and accidents will increase, leading to litigation and a reduction in the work we undertake.

Do I have evidence of this scenario? Recently, at a quayside near me, it was decided that it was necessary to separate cars from bikes from pedestrians from working areas outside. Up until this point, each individual was responsible for looking out for cars if on foot, for avoiding pedestrians if cycling, and so on. Over the period of a few days, the site was suddenly covered in swaths of red, green, and yellow paint separating everyone out in an ordered "safe" way. And, lest any mortal forget their designated color, large icons of bikes or people were added. People no longer had to think; the yellow (or red) brick road would lead them safely through life-brilliant! A couple of weeks after their colorful appearance, I encountered a group of students heading on their allotted path to a boat for a practical. At the time, a crane was lifting two tonnes of anchor chain from the quayside onto another vessel, across the red path. Without the red walkway, the students would have gone cautiously across a working area, observed the crane operation, and either waited or gone around the whole thing at a safe distance. They,

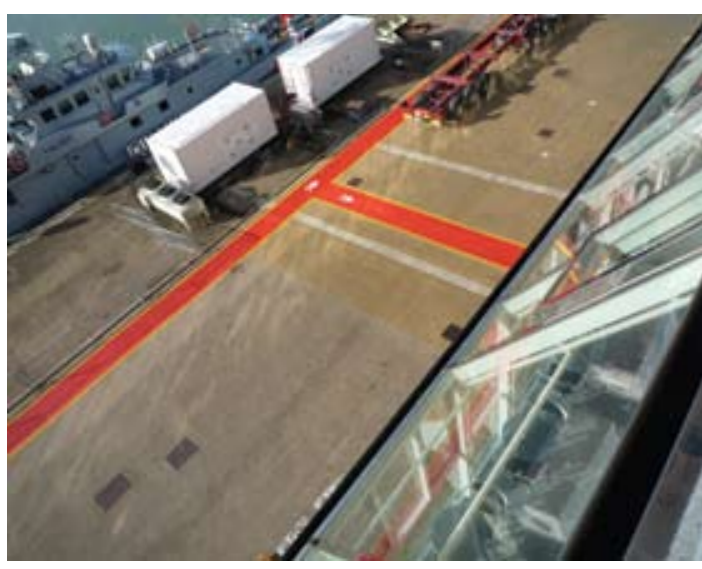

Figure 1. Red paths may not always lead to safety.

however, decided that they were on the pathway of safe deliverance and walked under the dangling chain, much to the horror of myself and the crane operator-even hard hats would have been of limited use here! When "questioned" by one irate me, they commented that they were on the safe path and shouldn't have to check around them anymore. Safety had been taken out of the duty of the individual. Had they been crushed, all would have been fine. After all, they were on the red path. I now wonder if that's why the powers that be had chosen red?

Safety is paramount to our work, but it has to be learned and experienced. Our students have to develop a sense of safe working and that does mean occasional falls or controlled mishaps. It is a problem that can only be solved by pragmatic approaches, particularly in the field of oceanography, which is inherently hazard filled. We need the paperwork-but we need the experience far more. 近

\section{REFERENCES}

Slovic, P. 1987. Perception of risk. Science 236:280-285. Cook, V.A., D. Phillips, and J. Holden. 2006. Geography fieldwork in a "risk society." Area 38(4):431-420. 